\title{
REPRESENTATION OF NUMBERS IN TERNARY QUADRATIC FORMS
}

\author{
E. ROSENTHALL
}

We employ integral quaternions $t=t_{0}+t_{1} i_{1}+t_{2} i_{2}+t_{3} i_{3}$, where the coordinates $t_{i}$ range over rational integers, while the $i_{1}, i_{2}, i_{3}$, satisfy the multiplication table

$$
\begin{aligned}
& i_{1}{ }^{2}=i_{2}{ }^{2}=-2, \quad i_{3}{ }^{2}=-3, \quad i_{2} i_{3}=2 i_{1}-i_{2}=\left(\overline{i_{3} i_{2}}\right), \\
& i_{3} i_{1}=-i_{1}+2 i_{2}=\left(\overline{i_{1} i_{3}}\right), \quad i_{1} i_{2}=-1+i_{3}=\left(\overline{i_{2} i_{1}}\right),
\end{aligned}
$$

and $\vec{t}=t_{0}-t_{1} i_{1}-t_{2} i_{2}-t_{3} i_{3}$ is the conjugate to $t$. The norm $N(t)$ of $t$ is $t \bar{t}=\bar{t} t=t_{0}^{2}+2 t_{1}^{2}+2 t_{2}^{2}+2 t_{1} t_{2}+3 t_{3}^{2}$. The norm of a product of two quaternions equals the product of their norms, and $\overline{v t}=\bar{t} \bar{v}$ for any two quaternions. The associative law $r s \cdot t=r \cdot s t$ holds.

The quaternary quadratic $Q=t_{0}^{2}+2 t_{1}{ }^{2}+2 t_{2}{ }^{2}+2 t_{1} t_{2}+3 t_{3}{ }^{2}$ has determinant 9 , the g.c.d. of the literal coefficients of the adjoint to $Q$ is 3 , and the second concomitant of $Q$ represents no residues 1 modulo 3, and as there is only one form of determinant 9 with these properties in Charve's table* of reduced quaternary quadratic forms, $Q$ belongs to a genus of one class. Since $Q$ represents 1 for two values of $t_{0}, \cdots, t_{3}$, we have, $\uparrow$ a proper quaternion being defined as one having coprime coordinates, the following theorem:

TheOREM 1. A proper quaternion $v=v_{0}+v_{1} i_{1}+v_{2} i_{2}+v_{3} i_{3}$ whose norm is divisible by a positive integer $m$ prime to 6 has exactly two rightdivisors (left-divisors) $t$ and $-t$ of norm $m$.

Every proper pure quaternion $s=s_{1} i_{1}+s_{2} i_{2}+s_{3} i_{3}$ of norm $\mathrm{km}^{2}$ is of form $\bar{t} a t$ where $N(a)=k$ and $N(t)=m$. For, $s=v t$ where $N(t)=m$ by Theorem $1 ; \bar{s}=-s=\bar{t} \bar{v}$, and $\bar{t}$ is a left-divisor of $s$. Hence, since $N(v)=k m, \bar{t}$ is a left-divisor of the proper quaternion $v, v=\bar{t} a$. Hence $s=\bar{t} a t, N(a)=k$. Clearly $a$ is pure since $\bar{t} \bar{a} t=-\bar{t} a t, \bar{a}=-a$.

Theorem 2. Consider the equation $24 n+1=x_{1}{ }^{2}+2 x_{2}{ }^{2}+2 x_{3}{ }^{2}-2 x_{2} x_{3}$. If $24 n+1=m^{2},(m>0)$, then all proper solutions are of type $A$ if $m \equiv 1$ $(\bmod 4)$ but of type $B$ if $m \equiv 3(\bmod 4)$, where

$$
A: x_{1} \equiv \pm 1(\bmod 12), \quad B: x_{1} \equiv \pm 5(\bmod 12) \text {. }
$$

* L. Charve, Comptes Rendus de l'Académie des Sciences, vol. 96 (1883), p. 773.

$\dagger$ G. Pall, On the factorization of generalized quaternions, submitted to the Duke Mathematical Journal. 
If $24 n+1$ is not a square, there are equally many solutions of each type $A$ and $B$.

A proof for the case in which $24 n+1=m^{2}$ follows. Consider

$$
h=x_{1}^{2}+2 x_{2}^{2}+2 x_{3}^{2}-2 x_{2} x_{3} .
$$

Then

$3 h=3 x_{1}^{2}+2\left(-x_{2}-x_{3}\right)^{2}+2\left(-x_{2}-x_{3}\right)\left(2 x_{2}-x_{3}\right)+2\left(2 x_{2}-x_{3}\right)^{2}$.

Put $h=m^{2}$, and let $x=i_{1}\left(-x_{2}-x_{3}\right)+i_{2}\left(2 x_{2}-x_{3}\right)+i_{3} x_{1}$ represent a solution $\left(x_{1}, x_{2}, x_{3}\right)$ of (1); then all proper pure quaternions $x$ are given by $x=\bar{t} a t, N(t)=m, N(a)=3$, and from the latter condition we must have $a= \pm i_{3}$. Expanding $\bar{t} a t$ gives $x_{1}=3 t_{3}{ }^{2}-2 t_{1}{ }^{2}-2 t_{2}{ }^{2}-2 t_{1} t_{2}+t_{0}{ }^{2}$ where only $a=i_{3}$ is considered since $a=-i_{3}$ merely changes the sign of $x_{1}$ which leaves $A$ and $B$ unaltered.

Thus $x_{1} \equiv m(\bmod 4)$. Since $(m, 3)=1, x_{1} \equiv 1(\bmod 3) ;$ hence when $m \equiv 1(\bmod 4), x_{1} \equiv \pm 1(\bmod 12)$, a solution of type $A$, but if $m \equiv 3$ $(\bmod 4)$, then $x_{1} \equiv \pm 5(\bmod 12)$, a solution of type $B$.

The case for $h=24 n+1$ not a square will now be considered. Let $x$ be a representative solution of (1) under this condition. We can choose an odd prime $p$ such that simultaneously

$$
(-3 h \mid p)=1, \quad p \equiv 11(\bmod 12) .
$$

By the first equation in (2) we can choose $x_{0}$ so that $3 x_{0}{ }^{2}+h \equiv 0$ $(\bmod p)$. Then by Theorem $1,3 x_{0}+x$ has exactly two right-divisors $\pm t$ of norm $p$, say

$$
3 x_{0}+x=u t, \quad N(t)=p .
$$

Then

$$
t x \bar{t}=p y, \quad \text { where } \quad y=t u-3 x_{0},
$$

and $y$ represents another solution of (1). If $t$ is replaced by $-t, y$ is unchanged.

We shall prove that $x$ and $y$ are in opposite classes $A$ and $B$ in view of the second equation of (2), and as multiplication by $p$ does not alter $A$ or $B$, it will suffice to show that $x$ and $t x \bar{t}$ are solutions of opposite types.

Setting $t x \bar{t}=i_{1}\left(-y_{2}-y_{3}\right)+i_{2}\left(2 y_{2}-y_{3}\right)+i_{3} y_{1}$ and expanding gives

$$
\begin{aligned}
y_{1}=x_{1}\left(t_{0}{ }^{2}+3 t_{3}{ }^{2}-2 t_{2}{ }^{2}-2 t_{1}{ }^{2}-\right. & \left.2 t_{1} t_{2}\right)+x_{2}\left(6 t_{2} t_{3}+4 t_{1} t_{0}+2 t_{2} t_{0}\right) \\
& +x_{3}\left(-6 t_{2} t_{3}-6 t_{3} t_{1}+2 t_{2} t_{0}-2 t_{1} t_{0}\right) .
\end{aligned}
$$

In (1), $x_{2}$ and $x_{3}$ are always even, and thus 


$$
y_{1} \equiv 3 x_{1}(\bmod 4), \quad y_{1} \equiv x_{1}(\bmod 3)
$$

as $N(t) \equiv 11(\bmod 12)$. Hence $y$ represents a solution of type opposite to $x$.

We can now establish the $(1,1)$ correspondence. We employ the preceding process for a fixed $p$, with $x_{0}$ for a solution of type $A$, but $-x_{0}$ for a solution of type $B$. Hence if our process carries $x$, a solution of type $A$, into $y$, then $y$ is carried into $x$. For from (3)

$$
3\left(-x_{0}\right)+y=(-\bar{u}) \bar{t}, \quad N(\bar{t})=p .
$$

Then, $\bar{t} y t=p x, x=u t-3 x_{0}$. Further, two distinct solutions of one type cannot correspond to the same solution of the other type.

Application of other types of quaternions furnishes arithmetical proofs of the following additional results:

For representation of $24 n+1$ in $x_{1}{ }^{2}+3 x_{2}^{2}+3 x_{3}^{2}$ the $A$ and $B$ relations are

$$
\begin{array}{r}
A: x_{1} \equiv \pm 1(\bmod 12), x_{2} \text { and } x_{3} \equiv 0(\bmod 4), \\
x_{1} \equiv \pm 5(\bmod 12), x_{2} \text { and } x_{3} \equiv 2(\bmod 4), \\
B: x_{1} \equiv \pm 1(\bmod 12), x_{2} \text { and } x_{3} \equiv 2(\bmod 4), \\
x_{1} \equiv \pm 5(\bmod 12), x_{2} \text { and } x_{3} \equiv 0(\bmod 4) .
\end{array}
$$

For $2(24 n+1)=3 x_{1}^{2}+x_{2}^{2}+x_{3}^{2}$

$$
\begin{aligned}
& A:(0 ; 1,1),(0 ; 7,7),(0 ; 11,5),(4 ; 5,5),(4 ; 11,11),(4 ; 7,1), \\
& B:(4 ; 1,1),(4 ; 7,7),(4 ; 11,5),(0 ; 5,5),(0 ; 11,11),(0 ; 7,1),
\end{aligned}
$$

where each triplet $\left(x_{1} ; x_{2}, x_{3}\right)$ lists the least absolute residues $x_{1}$ $(\bmod 8), x_{2}(\bmod 24), x_{3}(\bmod 24)$ in a definite order.

For either form if $24 n+1=m^{2},(m>0)$, all solutions are of type $A$ if $m \equiv 1(\bmod 6)$, but of type $B$ if $m \equiv 5(\bmod 6)$. But there are equally many solutions of each type if $24 n+1$ is not a square.

These results were proved in the writer's thesis at McGill University, 1938.

MCGILL UNIVERSITY 